\title{
Isolinderalactone enhances the inhibition of SOCS3 on STAT3 activity by decreasing miR-30c in breast cancer
}

\author{
MENG-CHI YEN ${ }^{1 *}$, YING-CHU SHIH ${ }^{2 *}$, YA-LING HSU ${ }^{2}$, EN-SHYH LIN ${ }^{3}$, YI-SHIUAN LIN ${ }^{4}$, \\ EING-MEI TSAI ${ }^{2}$, YA-WEN HO ${ }^{4}$, MING-FENG HOU ${ }^{4}$ and PO-LIN KUO ${ }^{2,4,5}$
}

\author{
${ }^{1}$ Department of Emergency Medicine, Kaohsiung Medical University Hospital, and ${ }^{2}$ Graduate Institute of Medicine, \\ College of Medicine, Kaohsiung Medical University, Kaohsiung 807; ${ }^{3}$ Department of Beauty Science, National Taichung \\ University of Science and Technology, Taichung 403; ${ }^{4}$ Institute of Clinical Medicine, College of Medicine, \\ Kaohsiung Medical University, Kaohsiung 807; Institute of Medical Science and Technology, \\ National Sun Yat-Sen University, Kaohsiung 804, Taiwan, R.O.C.
}

Received October 4, 2015; Accepted November 9, 2015

DOI: 10.3892/or.2015.4503

\begin{abstract}
Development of an efficient treatment for triplenegative breast cancer is an urgent issues. Compounds from plant extracts are a potential source of novel cancer treatment. Isolinderalactone, a kind of sesquiterpenoids compound, was purified from the root of Lindera strychnifolia and Neolitsea daibuensis and shows anti-inflammatory and anticancer capacity. In the present study, isolinderalactone induced apoptosis in MDA-MB-231 cells which is a kind of triple-negative breast cancer cell line through induction of an intrinsic mitochondria-mediated and caspase-independent cell death. Treatment of isolinderalactone increased the protein level of the suppressor of cytokine signaling 3 (SCOS3), decreased phosphorylation of the signal transducer and activator of transcription 3 (STAT3), and suppressed expression of the down-stream genes of the X-linked inhibitor of apoptosis protein in MDA-MB-231 cells. Our results further showed that the level of SOCS3 expression was induced by isolinderalactone due to inhibiting the microRNA hsa-miR30c-5p (miR-30c) expression. In addition, intraperitoneal injection of isolinderalactone induced apoptosis in a xenograft breast tumor while it did not significantly affect the histology of liver, kidney and lung of the treated mice. In conclusion, isolinderalactone induces apoptosis in MDA-MB-231 cells and suppresses STAT3 signaling pathway through regulation
\end{abstract}

Correspondence to: Professor Ming-Feng Hou or Professor Po-Lin Kuo, Institute of Clinical Medicine, College of Medicine, Kaohsiung Medical University, 100 Shih-Chuan 1st Road, Kaohsiung 804, Taiwan, R.O.C.

E-mail:mifeho@kmu.edu.tw

E-mail: kuopolin@seed.net.tw

*Contributed equally

Key words: isolinderalactone, breast cancer, suppressor of cytokine signaling 3, signal transducer and activator of transcription 3, miR-30c of SOCS3 and miR-30c. It may become a novel treatment for triple-negative breast cancer in the future.

\section{Introduction}

Breast cancer is the most common type of cancer and the second leading cause of cancer death in women (1). There are approximately $12-20 \%$ of triple-negative phenotypes (estrogen and progesterone receptors, Her2 negative) among breast cancer (2). Hormonal or Her2-trageted therapy treatment is not beneficial for patients with triple-negative breast cancer due to loss of these target receptors (3). Surgery and chemotherapy are the only available approaches for patients. However, the response of chemotherapy in triple-negative breast cancer is relatively poor (4). Therefore, development of effective treatment is still a critical issue for these breast cancers. The natural compound from traditional Chinese medicine is a potential source for identifying novel treatment.

The root of Lindera aggregata (SIMS) KOSTERM (synonym: Lindera strychnifolia) is a traditional herbal medicine in China (Wu Yao) and Japan (Uyaku) $(5,6)$. Various bioactivities including cytoxicity, improvement of insulin sensitivity, and slowing down the progression of diabetic nephropathy in diabetes mice were shown $(5,7,8)$. Isolinderalactone is one of known sesquiterpenes extracted from root tubers of L. aggregata (9). Wong et al observed iNOS inhibitory activity and anti-inflammatory activity of isolinderalactone among the secondary metabolites from roots of Neolitsea daibuensis (10). In addition, treatment of isolinderalactone induces apoptosis and inhibits proliferation in non-small cell lung cancer cell through Fas/Fas ligand pathway and cell cycle arrest (11). Above all, isolinderalactone revealed anti-inflammatory and anticancer capacity.

Recent evidence indicates that tumor development is associated with some inflammatory signaling pathways, such as signal transducer and activator of transcription (STAT) and Janus kinase (JAK) pathways (12). Furthermore, many types of natural compounds which showed anticancer capacity usually induced apoptosis (13). Since isolinderalactone could both suppress inflammation and induce apoptosis, we hypothesized 
isolinderalactone may be a potential treatment for triplenegative breast cancer.

In the present study, we determined whether isolinderalactone-induced apoptosis and inhibited growth in triple-negative breast tumor in vitro and in vivo. The mechanism of isolinderalactone-induced apoptosis and regulation of inflammatory signaling pathways were investigated.

\section{Materials and methods}

Cell lines and cell culture. The human breast cancer cells MDA-MB-231 and the human kidney epithelial cells HEK293T/17 were obtained from American Type Culture Collection (ATCC; Rockville, MD, USA). MDA-MB-231 was cultured in Leibovitz's L-15 medium and HEK293T/17 was cultured in Dulbecco's modified Eagle's medium. Both media were supplemented with $10 \%$ fetal bovine serum (FBS) and penicillin/streptomycin $(100 \mathrm{U} / 0.1 \mathrm{mg} / \mathrm{ml})$. All materials for cell culture were obtained from Invitrogen (Carlsbad, CA, USA).

Materials and antibodies. Isolinderalactone was purchased from ChemFaces (Wuhan, China) and was dissolved in dimethylsulfoxide (DMSO). Antibodies of Jak, phospho-Jak (Y1022/1023), cleaved PARP, signal transducer and activator of transcription 3 (STAT3), phospho-STAT3 (S727 and Y705), AIF, XIAP, EndoG, BCL-xL, SOCS3, Bax and $\alpha$-tubulin were obtained from Cell Signaling Technology. Anti-GAPDH antibody and anti-Lamin A/C antibody were obtained from Millipore and BD Transduction Laboratories, respectively.

Growth inhibition and clonogenic assay. Cell growth was evaluated by WST-1 assay (Clontech) according to the manufacturer's instructions. MDA-MB-231 was seeded into 96-well plates $\left(1 \times 10^{5}\right.$ cells/well) and incubated overnight for attachment. Cells were treated by vehicle ( $0.1 \%$ DMSO) or several concentrations of isolinderalactone in $100 \mu \mathrm{l}$ medium. Forty-eight hours after isolinderalactone treatment, the medium was replaced by WST-1 containing medium for $2 \mathrm{~h}$ incubation. The plate was read at a wavelength $450 \mathrm{~nm}$ using a PowerWave X340 microplate spectrophotometer (Bio-Tek Instruments Inc., Winooski, VT, USA). The percentage of growth inhibition was calculated using the following formula: [100 - $\left.\left(\mathrm{OD}_{\text {isolinderalactone treatment }} / \mathrm{OD}_{\text {vehicle control }}\right) \times 100 \%\right]$. OD is the abbreviation of optical density. To determine the long-term effect of isolinderalactone, 1,000 MDA-MB-231 cells were seeded into $6 \mathrm{~cm}$ dish and incubated overnight to attach. Cells were treated with isolinderalactone and vehicle control for $6 \mathrm{~h}$ and then incubated in fresh medium for 14 days (medium was replaced every 3-4 days). Colonies were stained with crystal violet $(0.4 \mathrm{~g} / \mathrm{l}$; Sigma) and the number of colonies were counted.

LDH assay. MDA-MB-231 cells were seeded into 96-well plates $\left(1 \times 10^{5}\right.$ cells/well $)$ and treated with isolinderalactone and vehicle control in $100 \mu \mathrm{l}$ medium. Medium was collected at $48 \mathrm{~h}$ after treatment and then was performed by LDH cytotoxicity detection kit (Clontech) according to the manufacturer's instruction. The plate was read at wavelength $492 \mathrm{~nm}$ using a PowerWave X340 microplate spectrophotometer. The
Table I. Primers.

\begin{tabular}{ll}
\hline Gene & \multicolumn{1}{c}{ Primer sequences } \\
\hline GAPDH & 5'-GAGTCAACGGATTTGGTCGT-3' \\
& 5'-TTGATTTTGGAGGGATCTCG-3' \\
SOCS3 & 5'-AGTCTGGGACCAAGAACCTG-3' \\
& 5'-CGGAGGAGGGTTCAGTAGGT-3' \\
hsa-miR-19a-3p & 5'-dTGTGCAAATCTATGCAAAACTGA-3' \\
hsa-miR-19b-3p & 5'-dTGTGCAAATCCCATGCAAAACTGA-3' \\
hsa-miR-30b-5p & 5'-dTGTAAACATCCTACACTCAGCT-3' \\
hsa-miR-30c-5p & 5'-dTGTAAACATCCTACACTCTCAGA-3' \\
hsa-miR-218-5p & 5'-dTTGTGCTTGATCTAACCATGT-3' \\
hsa-miR-203-3p & 5'-dGTGAAATGTTTAGGACCACTAG-3' \\
hsa-miR-650 & 5'-dAGGAGGCAGCGCTCTCAGGAC-3'
\end{tabular}

GAPDH, glyceraldehyde-3-phosphate dehydrogenase; SOCS3, suppressor of cytokine signaling 3 .

percentage of cytotoxicity is calculated by following formula: $\left[100 \% \times\left(\mathrm{OD}_{\text {isolinderalactone treatment }}-\mathrm{OD}_{\text {blank }}\right)-\left(\mathrm{OD}_{\text {vehicle }}-\mathrm{OD}_{\text {blank }}\right) /\right.$ $\left.\mathrm{OD}_{\text {vehicle }}-\mathrm{OD}_{\text {blank }}\right]$.

Annexin V staining. MDA-MB-231 was seeded into 6-well plates $\left(5 \times 10^{5}\right.$ cells/well) and treated with isolinderalactone and vehicle control for $48 \mathrm{~h}$. Cells were harvested and analyzed by Annexin V-FITC apoptosis detection kit (BD Biosciences). Stained cells were determined on a BD Accuri C6 flow cytometer and the results were analyzed by Accuri C6 software (both from BD Biosciences).

Fluorescent caspase activity assay. The caspase 9/6 and caspase 8 were measured using the ApoAlert caspase fluorescent assay kit (Clontech) according to the manufacturer's instruction. In brief, after treatment of MDA-MB-231 cells with isolinderalactone, cells were scraped and lysed in cell lysis buffer. Equivalent amount of cell lysates were mixed with reaction buffer and incubated at $37^{\circ} \mathrm{C}$ for $1 \mathrm{~h}$. The caspases $9 / 6$ catalyzed release fluorescence with an excitation wavelength of $380 \mathrm{~nm}$ and an emission wavelength of $460 \mathrm{~nm}$; the caspase 8 catalyzed release fluorescence with an excitation wavelength of $400 \mathrm{~nm}$ and an emission wavelength of $505 \mathrm{~nm}$. The results were monitored using a microplate reader (FLx800 Microplate Fluorescence Readers; Bio-Tek Instruments Inc.)

Fas ELISA assay. After isolinderalactone treatment, the cell lysate were collected and the level of Fas was measured by Human APO-1/FAS/CD95 ELISA kit (Novex, Invitrogen, Carlsbad, CA, USA) according to the manufacturer's instructions. The results were monitored the absorbance at $450 \mathrm{~nm}$ using a microplate reader (PowerWave X340).

Western blotting. After appropriate treatment in each experiment, cells were lysed in radioimmunoprecipitation assay buffer (RIPA) (Millipore) on ice for $30 \mathrm{~min}$. The total cell lysate was then collected after centrifugation at $4^{\circ} \mathrm{C}$, $12,000 \times \mathrm{g}$ for $15 \mathrm{~min}$. The nuclear protein was extracted by 
Table II. The sequence of SOCS3 3'UTR (from UCSC Genome Bioinformatics, https://genome.ucsc.edu/) and predictive binding site (underline).

Sequence of SOCS3 3'UTR

GGGGTAAAGGGCGCAAAGGGCATGGGTCGGGAGAGGGGACGCAGGCCCCTCTC

CTCCGTGGCACATGGCACAAGCACAAGAAGCCAACCAGGAGAGAGTCCTGTAGC TCTGGGGGGAAAGAGGGCGGACAGGCCCCTCCCTCTGCCCTCTCCCTGCAGAAT GTGGCAGGCGGACCTGGAATGTGTTGGAGGGAAGGGGGAGTACCACCTGAGTCT CCAGCTTCTCCGGAGGAGCCAGCTGTCCTGGTGGGACGATAGCAACCACAAGTG GATTCTCCTTCAATTCCTCAGCTTCCCCTCTGCCTCCAAACAGGGGACACTTCGGG AATGCTGAACTAATGAGAACTGCCAGGGAATCTTCAAACTTTCCAACGGAACTTG TTTGCTCTTTGATTTGGTTTAAACCTGAGCTGGTTGTGGAGCCTGGGAAAGGTGG AAGAGAGAGAGGTCCTGAGGGCCCCAGGGCTGCGGGCTGGCGAAGGAAATGGT CACACCCCCCGCCCACCCCAGGCGAGGATCCTGGTGACATGCTCCTCTCCCTGGC TCCGGGGAGAAGGGCTTGGGGTGACCTGAAGGGAACCATCCTGGTACCCCACAT CCTCTCCTCCGGGACAGTCACCGAAAACACAGGTTCCAAAGTCTACCTGGTGCCT GAGAGCCCAGGGCCCTTCCTCCGTTTTAAGGGGGAAGCAACATTTGGAGGGGAT GGATGGGCTGGTCAGCTGGTCTCCTTTTCCTACTCATACTATACCTTCCTGTACCT GGGTGGATGGAGCGGGAGGATGGAGGAGACGGGACATCTTTCACCTCAGGCTCC TGGTAGAGAAGACAGGGGATTCTACTCTGTGCCTCCTGACTATGTCTGGCTAAGA GATTCGCCTTAAATGCTCCCTGTCCCATGGAGAGGGACCCAGCATAGGAAAGCC ACATACTCAGCCTGGATGGGTGGAGAGGCTGAGGGACTCACTGGAGGGCACCAA GCCAGCCCACAGCCAGGGAAGTGGGGAGGGGGGGCGGAAACCCATGCCTCCCA GCTGAGCACTGGGAATGTCAGCCCAGTAAGTATTGGCCAGTCAGGCGCCTCGTG GTCAGAGCAGAGCCACCAGGTCCCACTGCCCCGAGCCCTGCACAGCCCTCCCTCC TGCCTGGGTGGGGGAGGCTGGAGGTCATTGGAGAGGCTGGACTGCTGCCACCCC GGGTGCTCCCGCTCTGCCATAGCACTGATCAGTGACAATTTACAGGAATGTAGCA GCGATGGAATTACCTGGAACAGTTTTTTGTTTTTGTTTTTGTTTTTGTTTTTGTGGG GGGGGGCAACTAAACAAACACAAAGTATTCTGTGTCAGGTATTGGGCTGGACAG GGCAGTTGTGTGTTGGGGTGGTTTTTTTCTCTATTTTTTTGTTTGTTTCTTGTTTTTT AATAATGTTTACAATCTGCCTCAATCACTCTGTCTTTTATAAAGATTCCACCTCCA GTCCTCTCTCCTCCCCCCTACTCAGGCCCTTGAGGCTATTAGGAGATGCTTGAAG AACTCAACAAAATCCCAATCCAAGTCAAACTTTGCACATATTTATATTTATATTC AGAAAAGAAACATTTCAGTAATTTATAATAAAGAGCACTATTTTTTAATGAAAAA

Nuclear Extract kit (Active Motif) according to the manufacturer's instructions. Equivalent amount of protein was loaded and separated by sodium dodecyl sulfate-polyacrylamide gel electrophoresis (SDS-PAGE) (6-12\%) and transferred to polyvinylidene difluoride membranes. The membrane was blocked in 5\% non-fat dry milk for $1 \mathrm{~h}$ and then incubated with each primary antibody overnight and peroxidase-conjugated secondary antibody for $1 \mathrm{~h}$. The results were detected using an enhanced chemiluminescence substrate (Millipore) on a imaging capture system (Alpha Innovation).

Quantitative real-time PCR analysis. Total RNA of isolinderalactone-treated MDA-MB-231 cells was extracted from TRIzol reagent (Invitrogen). Complementary DNA (cDNA) of mRNA was reverse transcribed by PrimeScript RT reagent kit and cDNA of microRNA (miRNA) by Mir-X miRNA First-Strand Synthesis kit (both from Clontech). The level of miRNA was determined on StepOne Plus Real-Time PCT System using Fast SYBR-Green Master Mix (both from Applied Biosystems). Primer sequences are listed in Table I.
miRNA transfection. The control miRNA mimic and hsa-miR30c-5p miRNA mimic were synthesized by Dharmacon. HEK-293T cells were seeded in a $6 \mathrm{~cm}$ dish and transfected with miRNA mimic by DharmaFECT transfection reagent (Dharmacon). Cells were harvested at 24 or $48 \mathrm{~h}$ for mRNA analysis and protein analysis, respectively.

Luciferase assay. The complete sequence of SOCS3 3'UTR is shown in Table II and was constructed into the pMirTarget vector (OriGene). The plasmid containing the putative binding sequence of miR-30c 'TATTTACA' is named as 'SOCS3 3'UTR'. The mutant miR-30c binding sequence 'TGCGCGCA' (SOCS3 3'UTR mutant) was achieved by QuikChange Lightning Site-Directed Mutagenesis kit (Agilent Technologies). For luciferase assay, $400 \mathrm{nM}$ of control miRNA or miR-30c mimics was transfected into HEK-293T cells in 24-well plates for $12 \mathrm{~h}$ and then the $0.8 \mu \mathrm{g}$ of pMirTarget vector, SOCS3 3'UTR or SOCS3 3'UTR mutant and $0.08 \mu \mathrm{g}$ of pRL Renilla Luciferase Control Reporter Vector (Promega) were co-transfected for $24 \mathrm{~h}$. Cells were harvested and 
A

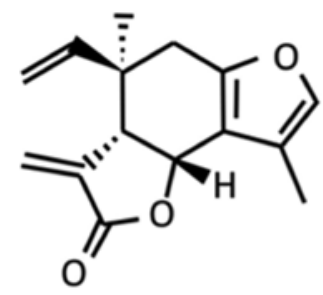

Isolinderalactone

C

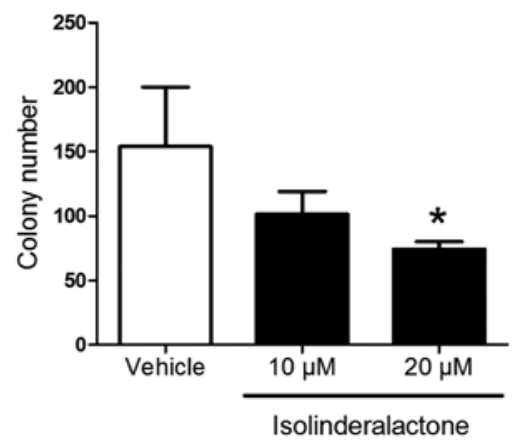

B

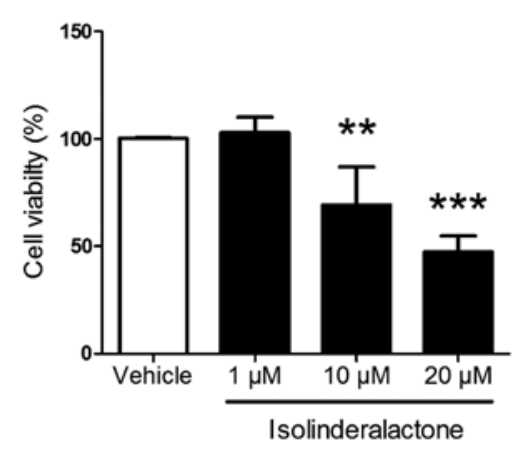

Figure 1. Isolinderalactone treatment inhibits breast cancer cell growth and colony formation. The MDA-MB-231 cells were treated with vehicle $(0.1 \%$ DMSO) and different dose of isolinderalactone. (A) The chemical structure of isolinderalactone. (B) The percentages of survival cells relative to vehicle control, defined as $100 \%$ survival were determined by WST-1 assay. (C) The number of colonies. Bars, mean \pm SEM. Statistically significant at ${ }^{* *} \mathrm{p}<0.01$; and ${ }^{* * * *} \mathrm{p}<0.001$. respectively. analyzed using the Dual-Luciferase Reporter Assay (Promega) according to the manufacturer's directions.

Animal experiments. The use of all the animals in the present study was approved by the Animal Care and Use Committee of the Kaohsiung Medical University. Six-weeks old male nude mice (BALB/cAnN.Cg-Foxn1 ${ }^{\text {nu}} / \mathrm{CrlNarl}$ ) were obtained from the National Laboratory Animal Center (Taiwan) and maintained in pathogen-free conditions. Mice were subcutaneously injected with $2 \times 10^{6}$ MDA-MB-231 cells in 200 microliter mixture of FBS-free L-15 medium/Matrigel at a ratio of 4/1. Treatment was initiated when the mean tumor volume reached $75 \mathrm{~mm}^{3}$. A total of 11 mice were randomly divided into two groups. The mice in the isolinderalactone-treated group were intraperitoneally daily injected with isolinderalactone at a dose of $10 \mathrm{mg} / \mathrm{kg}$ from an isolinderalactone solution ( $2 \mathrm{mg} / \mathrm{ml}$ ) containing $\sim 40 \%$ polyethylene glycerol, $60 \%$ PBS and $0.1 \%$ of DMSO. The control group was intraperitoneally injected daily with equal volume of PBS. Tumor volume was measured using calipers and tumor volume was calculated according to the formula: Length $(\mathrm{mm}) \mathrm{x}$ width $(\mathrm{mm}) \mathrm{x}$ width $(\mathrm{mm}) \times 1 / 2=$ tumor volume $\left(\mathrm{mm}^{3}\right)$. All mice were sacrificed when the mean tumor volume reached $1,000 \mathrm{~mm}^{3}$. The liver, kidney, and tumor were embedded in OCT for $5 \mu \mathrm{m}$ frozen section. General toxicity of liver and kidney was evaluated by hematoxylin and eosin (H\&E) staining. The apoptotic cells in tumors were detected by ApoAlert DNA Fragmentation Assay kit (Clontech) according to the manufacturer's instruction. 4',6-Diamidino-2-phenylindol (DAPI) was used as counterstain. The images were collected using a confocal microscope, LSM 700 (Carl Zeiss MicroImaging).

Statistical analysis. Difference between two independent groups were analyzed by the Student's t-test. Comparisons between three groups were performed using ANOVA with Dunnett's test. Significant difference $(p<0.05)$ between each group was considered. All calculations were carried out using the program GraphPad Prism version 5.03 (GraphPad Software, San Diego, CA, USA).

\section{Results}

Isolinderalactone treatment inhibits breast cancer cell growth and colony formation. A recent report indicates that isolinderalactone treatment inhibits cell proliferation and induces apoptosis in human non-small cell lung cancer cells (11). To further investigate the anti-tumor effect on triple-negative breast cancer, the triple-negative breast cancer cell line MDA-MB-231 was treated with different doses of isolinderalactone. The structure of isolinderalactone is shown in Fig. 1A. Isolinderalactone significantly inhibited cell viability and colony formation at the dose of $20 \mu \mathrm{M}$ (Fig. 1). The results indicated that isolinderalactone may serve as a novel treatment for triple-negative breast cancer.

Isolinderalactone treatment induces apoptosis in breast cancer cells. The result of lactate dehydrogenase (LDH) assay showed that the isolinderalactone treatment did not induce necrosis in MDA-MB-231 cells (Fig. 2A). The morphology of isolinderalactone-treated cells revealed the condensation of nuclear chromatin (Fig. 2B). In addition, isolinderalactone treatment increased the Annexin $\mathrm{V}$ - and PI-positive population and induced poly(ADP-ribose) polymerase (PARP) cleavage (Fig. 2C and D). The results indicated that isolinderalactone-induced apoptosis, but not necrosis in MDA-MB-231 cells.

Isolinderalactone treatment induces apoptosis in breast cancer cells through intrinsic mitochondria-mediated and 
A

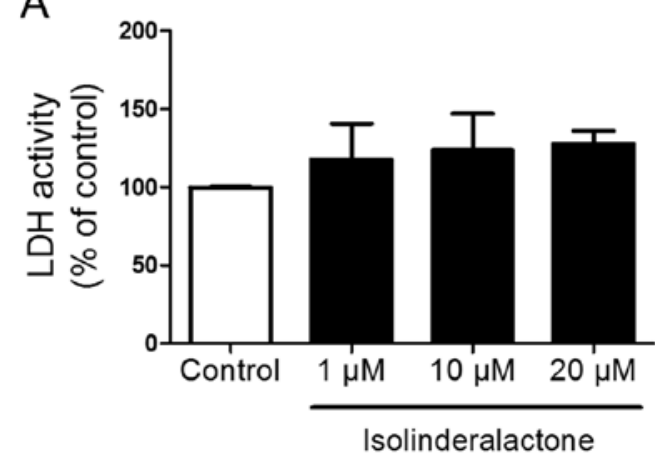

C

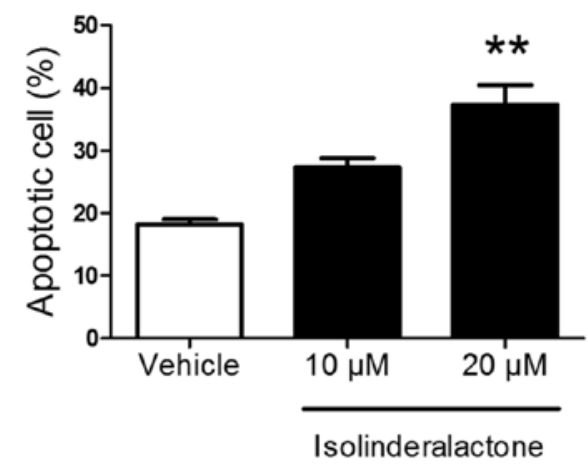

B

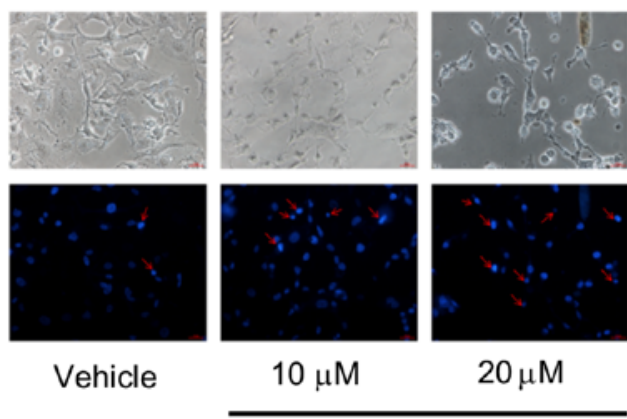

Isolinderalactone

D

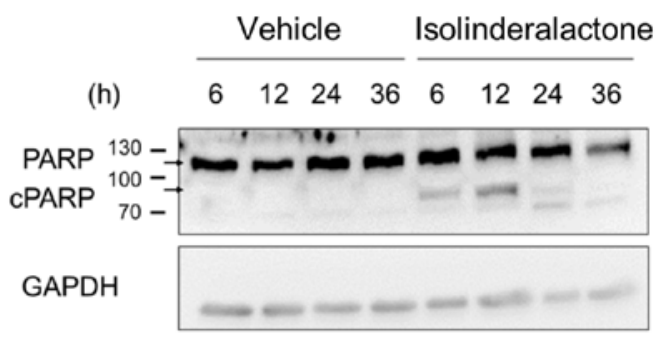

Figure 2. Isolinderalactone treatment induces apoptosis in breast cancer cells. (A) LDH activity assay. The released-LDH in medium of vehicle control was defined as $100 \%$. (B) Cell morphology after isolinderalactone treatment. The red arrow indicates the condensed chromatin in DAPI stained-MDA-MB-231 cells. (C) The apoptotic cells (including Annexin V- and PI-positive cells) were determined by Annexin V/PI staining. (D) MDA-MB-231 cells were treated with vehicle or $20 \mu \mathrm{M}$ isolinderalactone for $6,12,24$ and $36 \mathrm{~h}$. The level of cleaved PARP was detected by western blot analysis. Data are the representative of at least three independent experiments. Bars, mean \pm SEM. Statistically significant at ${ }^{* *} \mathrm{p}<0.01$.

caspase-independent cell death. Isolinderalactone treatment induced apoptosis in non-small cell lung cancer cells through Fas-mediated pathways (11). However, the Fas level was not affected by isolinderalactone treatment (Fig. 3A). We further determined the isolinderalactone-induced apoptosis pathways in breast cancer cells. Isolinderalactone treatment did not significantly induce either caspase 8 or 9 activity (Fig. 3B and C). Furthermore, reduced level of $\mathrm{Bcl}-\mathrm{xL}$ and increased level of Bax were detected after isolinderalactone treatment (Fig. 3D). In addition, increased level of apoptosis-inducing factor (AIF) and endonuclease $\mathrm{G}$ (EndoG) were observed in the nucleus (Fig. 3E). The results suggest that isolinderalactone treatment induced intrinsic mitochondriamediated and caspase-independent cell death.

Isolinderalactone treatment suppresses phosphorylation of STAT3 through SOCS3-dependent pathway. JAK-STAT3 signaling pathways are associated with cell differentiation, apoptosis and inflammation. Furthermore, activation of STAT3 signaling induces surviving gene expression and resists apoptosis in human breast cancer cells $(14,15)$. Fig. 4A and B, shows that the level of JAK1, phosphorylated-JAK1 and STAT3 was not affected by isolinderalactone. However, the level of phospho-STAT3 (Ser727) was suppressed in $3 \mathrm{~h}$ treatment. Decreased level of the molecule X chromosome-linked inhibitor of apoptosis (XIAP) was detected after treatment (Fig. 4C). The results indicated that STAT3 signaling was suppressed by isolinderalactone treatment. Suppressor of the cytokine signaling 3 (SOCS3) is a repressor of STAT3 signaling pathway (16). The induction of SOCS3 was observed at similar time point with downregulation of STAT3 phosphorylation after treatment (Fig. 4D). It may imply that the STAT3 repression is mediated through SOCS3 induction. In addition, the result may suggest isolinderalactone induces SOCS3 expression.

Isolinderalactone treatment enhances SOCS3 expression through inhibition of miR-30c expression. In various types of cancer, several kinds of micro-RNA (miRNA) regulate STAT3 signaling pathway through inhibition of SOCS3 expression (17-19). In order to investigate whether isolinderalactone treatment induce SOCS3 through regulation of miRNA, the miRNAs which targeted the 3' untranslated region (3'UTR) of SOCS3 were predicted by TargetScan website (v6.2; http:// targetscan.org) $(20,21)$. The level of miRNAs which may target on SOCS3 was analyzed by Q-PCR after treatment. Isolinderalactone treatment significantly suppressed the level of the hsa-miR30c-5p (miR-30c), but did not affect other miRNAs (Fig. 5A). The reporter plasmids which contain wildtype SOCS3 3'UTR, or putative miR-30c binding site mutated SOCS3 3'UTR were constructed for determining the binding capacity of miR-30c. The reporter assay demonstrated that miR-30c could bind the 3'UTR region of SOCS3 (Fig. 5B). Overexpression of miR-30c decrease the protein level of SOCS3 but did not affect the mRNA level of SOCS3 (Fig. 5C and D). The results suggested that regulation of SOCS3 by miR-30c was mainly through suppression of translation efficiency. 
A

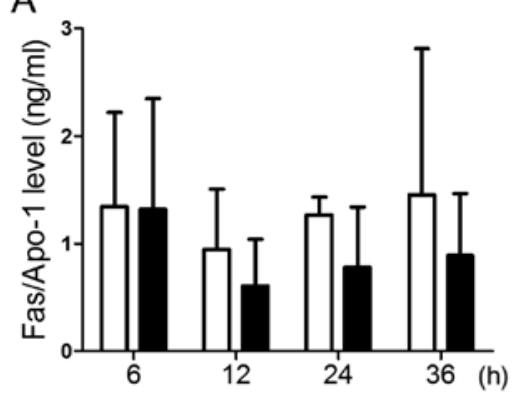

C

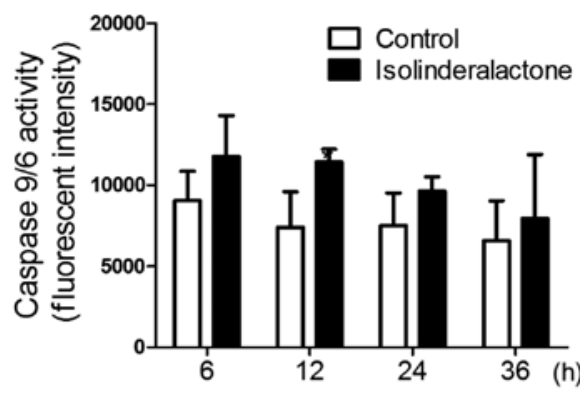

B

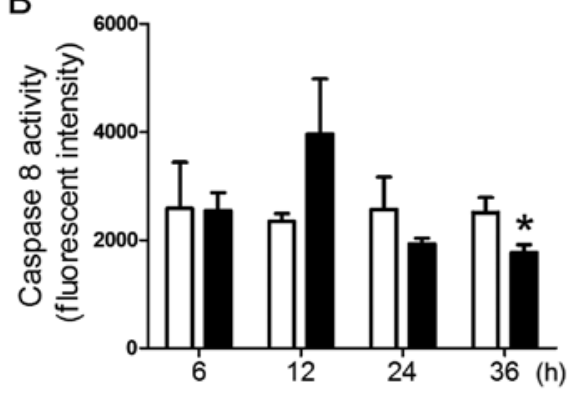

D (h) $\frac{6}{-+} \frac{12}{-+} \frac{24}{-++} \frac{36}{-+}$ Bcl-xL

Bax

$\alpha$-tubulin

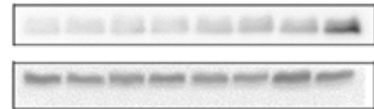

E

Control Isolinderalactone

$\begin{array}{llllllll}6 & 12 & 24 & 36 & 6 & 12 & 24 & 36\end{array}$

EndoG

AIF

- - - - -

$\operatorname{Lamin} \mathrm{A} / \mathrm{C}$

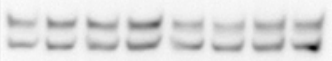

Figure 3. Isolinderalactone treatment induces apoptosis in breast cancer cells through intrinsic mitochondria-mediated and caspase-independent cell death. MDA-MB-231 cells were treated with vehicle or $20 \mu \mathrm{M}$ isolinderalactone for 6, 12, 24 and $36 \mathrm{~h}$. (A) Detection of Fas level in supernatant. (B) Detection of relative caspase 8 activity. (C) Detection of relative caspase 9/6 activity. (D) The expression level of Bcl-xL and Bax. (E) The expression level of EndoG and AIF. Data are representative of at least three independent experiments. Bars, mean \pm SEM. Statistically significant at ${ }^{*} \mathrm{p}<0.05$.

A

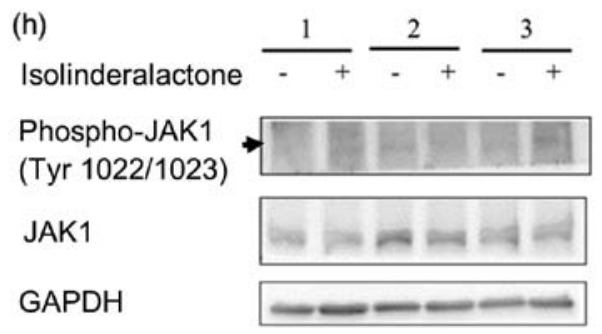

C

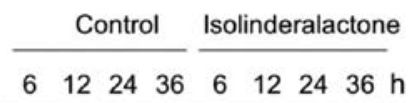

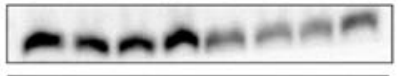

GAPDH

B

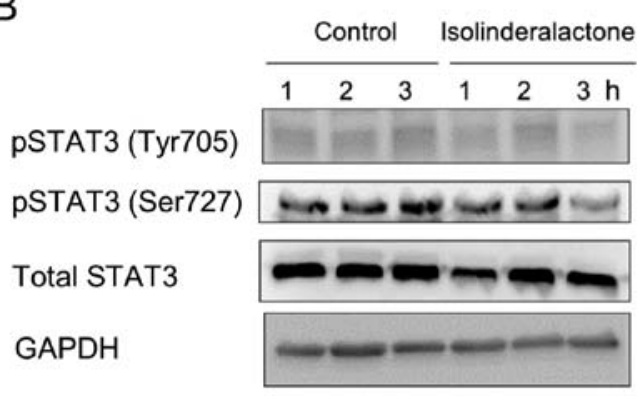

D

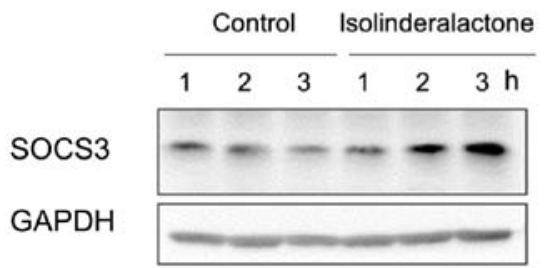

Figure 4. Isolinderalactone treatment suppresses phosphorylation of STAT3 through SOCS3-dependent pathway. MDA-MB-231 cells were treated with vehicle or $20 \mu \mathrm{M}$ isolinderalactone at different time points. The expression was analyzed by western blotting. (A) The expression level of JAK1 and phosphorylatedJAK1. (B) The expression level of STAT3 and phosphorylated-STAT3. (C) The expression of SOCS3. (D) The expression of XIAP. Data are representative of at least three independent experiments. 
A

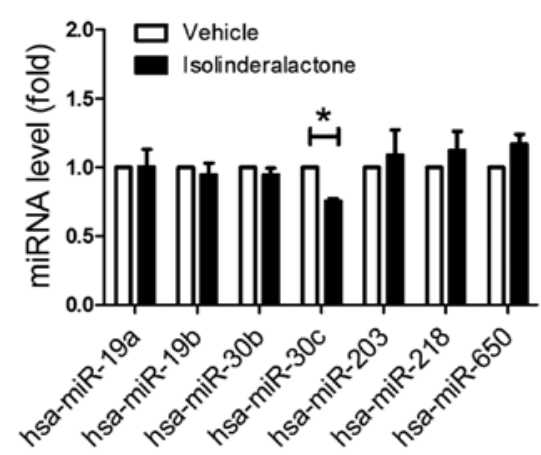

C

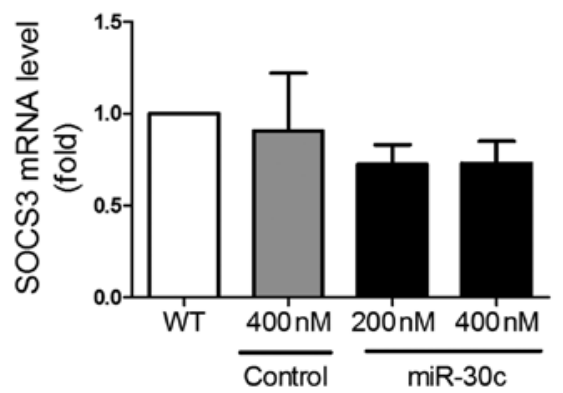

B

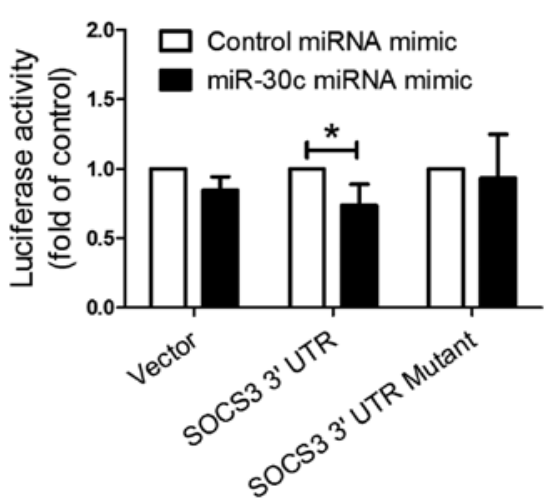

D

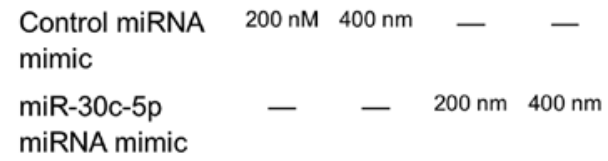

$\operatorname{socs} 3$

GAPDH

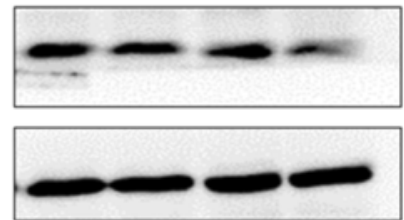

Figure 5. Isolinderalactone treatment enhances SOCS3 expression through inhibition of hsa-miR-30c expression. (A) MDA-MB-231 cells were treated with vehicle or $20 \mu \mathrm{M}$ isolinderalactone for $30 \mathrm{~min}$. The level of each miRNA was determined by Q-PCR. (B) HEK-293T cells were transfected with control miRNA mimic or miRNA mimics for miR-30c for $12 \mathrm{~h}$, and were then transfected with reporter plasmid including control luciferase expressing-vector (vector), containing 3'UTR region of the SOCS3 mRNA (SOCS3 3'UTR), and vector containing the mutant miR-30c binding site of 3'UTR region of the SOCS3 mRNA (SCOS3 3'UTR mutant) for $24 \mathrm{~h}$. Relative luciferase activity is shown. (C) HEK-293T cells were transfected with control miRNA mimic or miR-30c miRNA mimic for $24 \mathrm{~h}$. The level of miRNA was detected by Q-PCR. (D) HEK-293T cells were transfected with control miRNA mimic or miRNA mimic for hsa-miR-30c with the concentration as indicated for $48 \mathrm{~h}$. Cells were harvested for western blot assay. Bars, mean \pm SEM. Statistically significant at ${ }^{*} \mathrm{p}<0.05$.

Isolinderalactone treatment inhibits tumor growth and induces apoptosis in a xenograft breast tumor model. We further investigated whether isolinderalactone treatment inhibited tumor growth in the animal tumor model. In the xenograft tumor model, the tumor size and tumor weight of the isolinderalactone treated-mice is smaller than that of vehicle-treated mice (Fig. 6A and B). Isolinderalactone treatment also induced apoptosis and SOCS3 expression in the tumor (Fig. 6C and D). Finally, we examined whether isolinderalactone induced toxicity on liver, kidney and lung of mice. No significant histology between vehicle and isolinderalactone treatment groups was noted (Fig. 6E). Isolinderalactone induced only slight hypokalemic nephropathy. The results indicated isolinderalactone treatment suppressed tumor growth, induced apoptosis and SOCS3 expression without significant toxicity in mice.

\section{Discussion}

Isolinderalactone treatment inhibited proliferation and colony formation in MDA-MB-231 cells (Fig. 1). When the cells were treated with a dose of $20 \mu \mathrm{M}$, apoptosis was significantly induced (Fig. 2). Induction of apoptosis could be through intrinsic and extrinsic pathways (22). In extrinsic pathway, the level of Fas was not affected after isolinderalactone treatment. Besides, caspase 8 activity was not significantly induced.
The results suggested that the apoptosis was via the intrinsic pathway. Although isolinderalactone treatment did not increase the activity of caspase 9, it increased the level of Bcl-xL and decreased the level of Bax. Therefore, the isolinderalactoneinduced apoptosis may be through caspase 9-independent intrinsic apoptosis pathway (23). AIF and EndoG are mitochondrial pro-apoptotic proteins and translocate to the nucleus during mitochondria-mediated apoptosis $(24,25)$. The level of AIF and EndoG was significantly induced in the nucleus after treatment. The results suggest that isolinderalactone treatment cause intrinsic mitochondria-mediated and caspase-independent cell death in MDA-MB-231 cells.

We demonstrated that isolinderalactone treatment inhibited the phosphorylation of STAT3 and STAT3 signaling pathway. In Fig. 4C, the decreased XIAP protein level was observed in treated cells since XIAP is an inhibitor of apoptosis and a downstream target for STAT3 activation $(26,27)$. In addition, the oncogenic STAT3 activation increased expression of prosurvival proteins including survivin, Bcl-2 and Bcl-xL (15). Thus, the decreased Bcl-xL protein level was detected in Fig. 3D. The evidence indicates that STAT3 plays an important role in isolinderalactone-mediated apoptosis.

SOCS3 is a repressor of STAT3 signaling pathway. In addition, SOCS3 plays a negative feedback regulator of STAT3 (16). In the present study, the induction of SOCS3 and the repression of STAT3 phosphorylation was observed at similar time point 
A

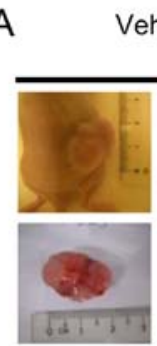

Vehicle

Isolinderalactone

C

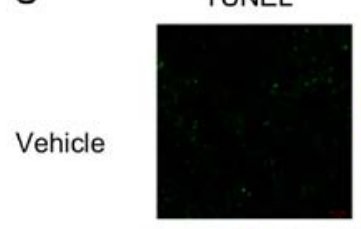

Isolinderalactone

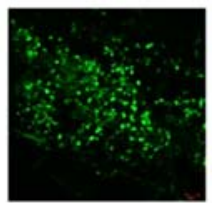

E
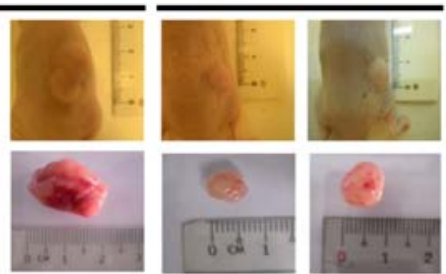

DAPI
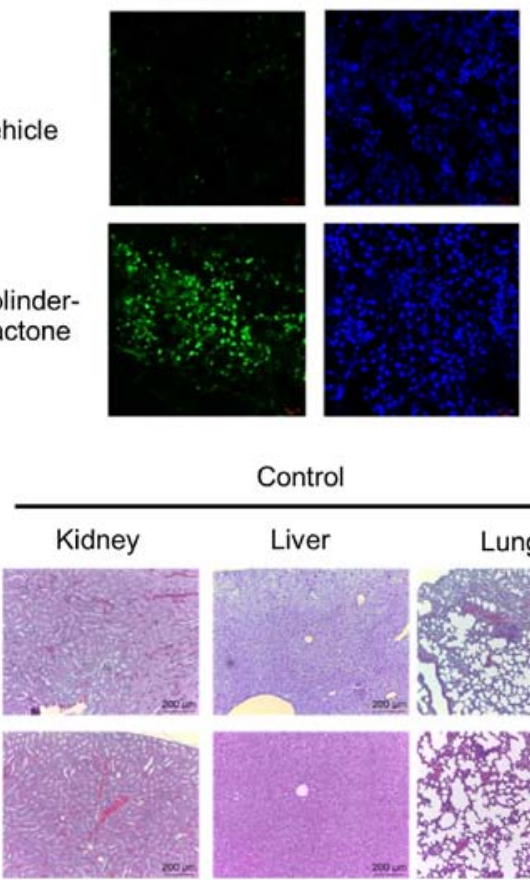

Control

Liver

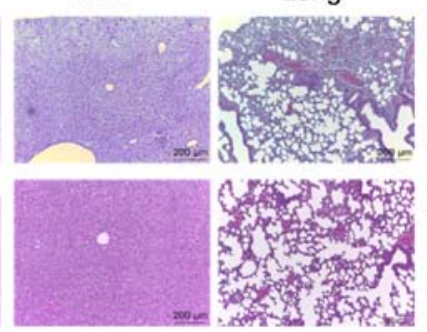

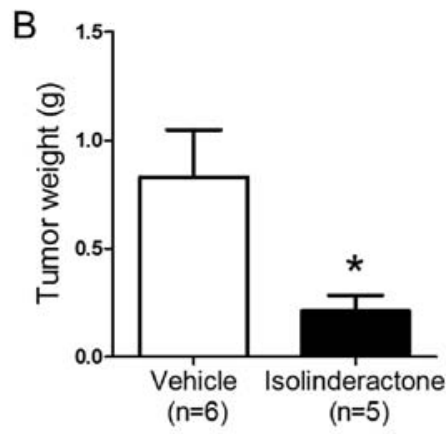

D

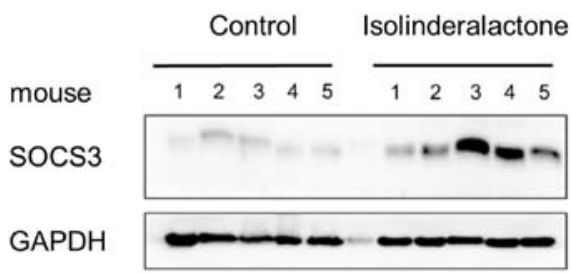

Isolinderalactone

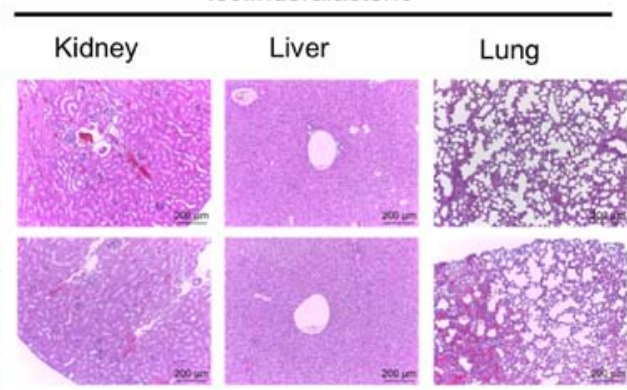

Figure 6. Isolinderalactone treatment inhibits tumor growth and induces apoptosis in a xenograft breast tumor model. Mice were subcutaneously injected with $2 \times 10^{6}$ MDA-MB-231 cells. When the mean tumor volume reach $75 \mathrm{~mm}^{3}$, mice were intraperitoneally injected with vehicle and isolinderalactone $(10 \mathrm{mg} / \mathrm{kg} /$ day). Mice were sacrificed at day 27 after isolinderalactone treatment. (A) Tumor size. Images of the two mice are representative of the other mice. (B) Mean tumor weight. Bars: mean \pm SEM. Statistically significant at "p<0.05. (C) TUNEL and DAPI staining. (D) Western blot analysis for SOCS3 expression in tumor. The number indicates samples that were collected from five mice. (E) $\mathrm{H} \& \mathrm{E}$ staining for kidney, liver and lung from vehicle or isolinderalactone-treated mice. One representative result of at least five mice is shown.

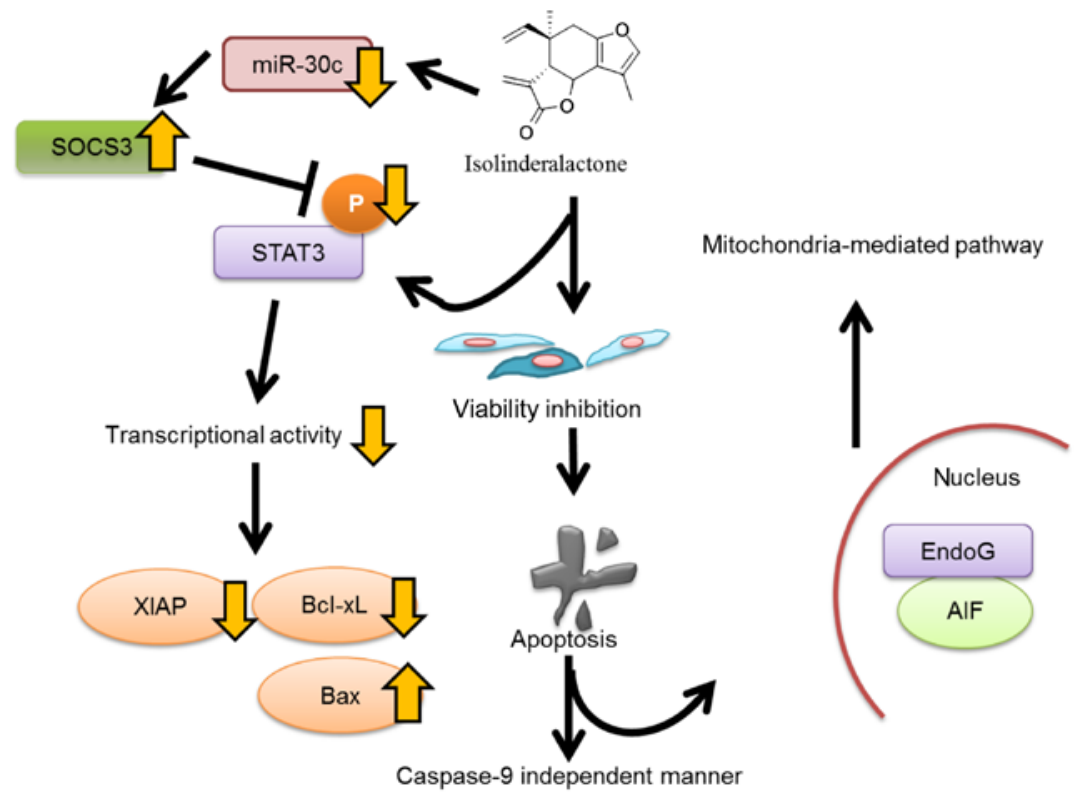

Figure 7. Isolinderalactone-induced pathways in MDA-MB-231 triple-negative breast cancer cells. 
after isolinderalactone treatment (Fig. 4B and D). It may suggest upregulation of SOCS3 is not regulated by negative feedback pathway of STAT3, but isolinderalactone treatment. Several studies indicated miRNAs such as miR-19b and miR-221 are involved in regulating SOCS3-mediated repression of STAT3 activation (17-19). After screening all putative miRNA targeting SOCS3 3'UTR, miR-30c was the only miRNA which was affected by isolinderalactone. Micro-RNAs regulate target gene expression through messenger RNA (mRNA) degradation and translation control (28). In the present study, our results further suggest that miR-30c targeted SOCS3 3'UTR and interfered in translation efficiency of SOCS3 (Fig. 5).

Isolinderalactone induced apoptosis and SOCS3 expression not only in vitro but also in vivo. In addition, no significant toxicity was observed in lung, liver and kidney of isolinderalactone-treated mice. In summary, isolinderalactone inhibits STAT3 activation since SOCS3 expression is enhanced by decreasing miR-30c expression in MDA-MB-231 cells (Fig. 7). It shows promise in a future treatment for triple-negative breast cancer.

\section{Acknowledgements}

The present study was supported by grants from the National Science Council (NSC; 102-2628-B-037-002-MY3, 102-2632-B-037-001-MY3 and 102-2314-B-037-035-MY3), the Ministry of Science and Technology (MOST; 104-2320-B-037-014-MY3, 104-2314-B-037-053-MY4 and 103-2320-B-037-006-MY3), and the Kaohsiung Medical University 'Aim for the Top 500 Universities Grant' (grant no. KMU-DT105010).

\section{References}

1. Siegel RL, Miller KD and Jemal A: Cancer statistics, 2015. CA Cancer J Clin 65: 5-29, 2015.

2. Anders CK and Carey LA: Biology, metastatic patterns, and treatment of patients with triple-negative breast cancer. Clin Breast Cancer 9 (Suppl 2): S73-S81, 2009.

3. Wahba HA and El-Hadaad HA: Current approaches in treatment of triple-negative breast cancer. Cancer Biol Med 12: 106-116, 2015.

4. Ismail-Khan R and Bui MM: A review of triple-negative breast cancer. Cancer Control 17: 173-176, 2010.

5. Ohno T, Nagatsu A, Nakagawa M, Inoue M, Li YM, Minatoguchi S, Mizukami H and Fujiwara H: New sesquiterpene lactones from water extract of the root of Lindera strychnifolia with cytotoxicity against the human small cell lung cancer cell, SBC-3. Tetrahedron Lett 46: 8657-8660, 2005.

6. Wang F, Gao Y, Zhang L, Bai B, Hu YN, Dong ZJ, Zhai QW, Zhu HJ and Liu JK: A pair of windmill-shaped enantiomers from Lindera aggregata with activity toward improvement of insulin sensitivity. Org Lett 12: 3196-3199, 2010.

7. Wang F, Gao Y, Zhang L and Liu JK: Bi-linderone, a highly modified methyl-linderone dimer from Lindera aggregata with activity toward improvement of insulin sensitivity in vitro. Org Lett 12: 2354-2357, 2010.

8. Ohno T, Takemura G, Murata I, Kagawa T, Akao S, Minatoguchi S, Fujiwara $\mathrm{T}$ and Fujiwara $\mathrm{H}$ : Water extract of the root of Lindera strychnifolia slows down the progression of diabetic nephropathy in $d b / d b$ mice. Life Sci 77: 1391-1403, 2005.

9. Kobayashi W, Miyase T, Sano M, Umehara K, Warashina T and Noguchi H: Prolyl endopeptidase inhibitors from the roots of Lindera strychnifolia F. Vill. Biol Pharm Bull 25: 1049-1052, 2002.
10. Wong SL, Chang HS, Wang GJ, Chiang MY, Huang HY, Chen $\mathrm{CH}$, Tsai SC, Lin CH and Chen IS: Secondary metabolites from the roots of Neolitsea daibuensis and their anti-inflammatory activity. J Nat Prod 74: 2489-2496, 2011.

11. Chang WA, Lin ES, Tsai MJ, Huang MS and Kuo PL: Isolinderalactone inhibits proliferation of A549 human non-small cell lung cancer cells by arresting the cell cycle at the $\mathrm{G}_{0} / \mathrm{G}_{1}$ phase and inducing a Fas receptor and soluble Fas ligandmediated apoptotic pathway. Mol Med Rep 9: 1653-1659, 2014.

12. Yu H, Pardoll D and Jove R: STATs in cancer inflammation and immunity: A leading role for STAT3. Nat Rev Cancer 9: 798-809, 2009.

13. Wang CY, Bai XY and Wang CH: Traditional Chinese medicine: A treasured natural resource of anticancer drug research and development. Am J Chin Med 42: 543-559, 2014.

14. Gritsko T, Williams A, Turkson J, Kaneko S, Bowman T, Huang M, Nam S, Eweis I, Diaz N, Sullivan D, et al: Persistent activation of stat 3 signaling induces survivin gene expression and confers resistance to apoptosis in human breast cancer cells. Clin Cancer Res 12: 11-19, 2006.

15. Song H, Wang R, Wang S and Lin J: A low-molecular-weight compound discovered through virtual database screening inhibits Stat 3 function in breast cancer cells. Proc Natl Acad Sci USA 102: 4700-4705, 2005.

16. White CA and Nicola NA: SOCS3: An essential physiological inhibitor of signaling by interleukin- 6 and G-CSF family cytokines. JAK-STAT 2: e25045, 2013.

17. Collins AS, McCoy CE, Lloyd AT, O'Farrelly C and Stevenson NJ: miR-19a: An effective regulator of SOCS3 and enhancer of JAK-STAT signalling. PLoS One 8: e69090, 2013.

18. Kneitz B, Krebs M, Kalogirou C, Schubert M, Joniau S, van Poppel H, Lerut E, Kneitz S, Scholz CJ, Ströbel P, et al: Survival in patients with high-risk prostate cancer is predicted by miR-221, which regulates proliferation, apoptosis, and invasion of prostate cancer cells by inhibiting IRF2 and SOCS3. Cancer Res 74: 2591-2603, 2014.

19. Patel K, Kollory A, Takashima A, Sarkar S, Faller DV and Ghosh SK: MicroRNA let-7 downregulates STAT3 phosphorylation in pancreatic cancer cells by increasing SOCS3 expression. Cancer Lett 347: 54-64, 2014.

20. Agarwal V, Bell GW, Nam JW and Bartel DP: Predicting effective microRNA target sites in mammalian mRNAs. Elife 4: 4, 2015.

21. Garcia DM, Baek D, Shin C, Bell GW, Grimson A and Bartel DP: Weak seed-pairing stability and high target-site abundance decrease the proficiency of $l s y-6$ and other microRNAs. Nat Struct Mol Biol 18: 1139-1146, 2011.

22. Peter ME: Programmed cell death: Apoptosis meets necrosis. Nature 471: 310-312, 2011.

23. Arnoult D, Gaume B, Karbowski M, Sharpe JC, Cecconi F and Youle RJ: Mitochondrial release of AIF and EndoG requires caspase activation downstream of Bax/Bak-mediated permeabilization. EMBO J 22: 4385-4399, 2003.

24. Joza N, Susin SA, Daugas E, Stanford WL, Cho SK, Li CY, Sasaki T, Elia AJ, Cheng HY, Ravagnan L, et al: Essential role of the mitochondrial apoptosis-inducing factor in programmed cell death. Nature 410: 549-554, 2001.

25. Li LY, Luo $X$ and Wang $X$ : Endonuclease $G$ is an apoptotic DNase when released from mitochondria. Nature 412: 95-99, 2001.

26. Takahashi R, Deveraux Q, Tamm I, Welsh K, Assa-Munt N, Salvesen GS and Reed JC: A single BIR domain of XIAP sufficient for inhibiting caspases. J Biol Chem 273: 7787-7790, 1998.

27. Obexer $P$ and Ausserlechner MJ: X-linked inhibitor of apoptosis protein - a critical death resistance regulator and therapeutic target for personalized cancer therapy. Front Oncol 4: 197, 2014.

28. Valencia-Sanchez MA, Liu J, Hannon GJ and Parker R: Control of translation and mRNA degradation by miRNAs and siRNAs. Genes Dev 20: 515-524, 2006. 